\title{
Caracterización de la carne de cuy (Cavia porcellus) para utilizarla en la elaboración de un embutido fermentado
}

\section{Characterization of the guinea pig (Cavia porcellus) meat for fermented sausage preparation}

Fecha de recepción: 12 de febrero de 2016 Fecha de aprobación: 27 de abril de 2016

\author{
César Iván Flores-Mancheno' \\ Cira Duarte ${ }^{2}$ \\ Iván Patricio Salgado-Tello ${ }^{3}$
}

\begin{abstract}
Resumen
La carne de cuy, por su alto valor nutritivo, es importante en la alimentación humana, y clave para la seguridad alimentaria; además, es utilizada para la elaboración de productos agroindustriales. Las personas que consumen esta carne son menos propensas a enfermedades. El objetivo de la investigación fue caracterizar la carne de cuy para su posible utilización en la elaboración de un embutido fermentado. Las líneas de cuyes evaluadas fueron la Criolla, la Andina y la Peruana mejorada. La carne se caracterizó utilizando análisis de varianza para las diferencias y comparación de medias, según Duncan $(p<0.05)$, desde el punto de vista físico-químico, microbiológico y sensorial. El contenido de humedad no registró diferencias entre las tres líneas evaluadas (75,6 \% en el Andino, 73,3\% en el Peruano mejorado y 72,7 $\%$ en el Criollo). El mayor contenido de proteína (19,1\%) se encontró en el Criollo, así como el menor contenido de grasa $(7,6 \%)$. En cuanto a las características microbiológicas y sensoriales de la carne, cumple con los requisitos de calidad establecidos por las normas respectivas. La calidad de la carne de cuy permite su utilización en la formulación de un producto cárnico.
\end{abstract}

Palabras clave: Cavia porcellus; cuy; cuy andino; cuy criollo; cuy peruano mejorado.

\begin{abstract}
Guinea pig meat, due to its high nutritional value, is important in the human diet, and is a key product for food safety; additionally, this meat is used in the agroindustry production, and people who consume it are less prone to diseases. The objective of the investigation was to characterize the guinea pig meat, for its

1 Ph.D. Escuela Superior Politécnica de Chimborazo (Riobamba, Ecuador). c_flores@espoch.edu.ec.

2 Ph.D. Instituto de Investigaciones para la Industria Alimenticia (La Habana, Cuba). cira@iiia.edu.cu.

3 Ph.D. Escuela Superior Politécnica de Chimborazo (Riobamba, Ecuador).
\end{abstract}


possible use in the elaboration of an improved fermented sausage. The lines of guinea pig evaluated were the Creole, Andean and Peruvian.

Meat was characterized using analysis of variance for differences and means comparison, according to Duncan $(p<0,05)$, from a physicochemical, microbiological and sensorial point of view. The moisture content did not show differences between the three evaluated lines, whose values were $75.6 \%$ in the Andean, $73.3 \%$ in the improved Peruvian, and $72.7 \%$ in the Creole. The highest protein content was $19.1 \%$ in the Creole, which also had the lowest fat content $(7.6 \%)$. In regard to the meat microbiological and sensorial characteristics, it complied with the quality requirements established by the respective standards. The guinea pig meat quality allows its use in a meat product formulation.

Keywords: Cavia porcellus; guinea pig; guinea pig andean; guinea pig creole; improved peruvian.

\section{Cómo citar este artículo:}

Flores-Mancheno Cl, Duarte C, Salgado-Tello IP. Caracterización de la carne de cuy (Cavia porcellus) para utilizarla en la elaboración de un embutido fermentado. Rev. Cien. Agri. 2017; 14(1): 39-45. 


\section{Introducción}

El cuy (Cavia porcellus), conocido también como cobayo, curiel o curí, es un mamífero roedor que fue domesticado en la región Andina de Sudamérica, donde ha sido utilizado, principalmente, como fuente de alimento, y que ha tomado mucha fuerza en el mercado gastronómico internacional en forma de plato típico exótico, entre otros usos (1). Los cuyes, además, tienen un valor cultural espiritual y son utilizados como animal de exhibición o compañía (1). La carne de cuy es magra; con un contenido de grasa menor al $10 \%$, alto contenido de proteínas y bajo en colesterol y sodio es ideal para incluirla en una alimentación variada y equilibrada apta para todos los grupos poblacionales, desde niños hasta ancianos, y en diversas situaciones fisiológicas, como, por ejemplo, el embarazo o la lactancia (2). Esta carne es consumida principalmente como plato típico asado o en locro (guiso) en países como Perú, Ecuador, Colombia y Bolivia, ya que es muy sabrosa, suave, con alta calidad nutritiva y de fácil digestión (3); presenta una serie de beneficios para la salud humana, ya que es fuente de proteína, hierro y vitamina $B_{12}$, razón que la posiciona como un excelente alimento dietético (4). En Cuba no había tradición de criar cuyes con fines comestibles; sin embargo, por la necesidad de diversificar las fuentes de alimentación humana y aprovechar los beneficios de esta especie se introdujeron, en el año de 1992, estirpes mejoradas: primero, ejemplares del tipo Peruano, y posteriormente, el tipo Macabeo, originario de Ecuador (5). De acuerdo con el reglamento Técnico Ecuatoriano (6), la carne de cuy se clasifica como Tipo I, ya que su contenido de grasa es inferior al $15 \%$ y el de proteína es superior al $14 \%$.

Este trabajo de investigación tuvo como objetivo caracterizar la carne de cuy para su utilización en la elaboración de un embutido fermentado, y permitió obtener un nuevo producto cárnico fermentado de valor nutricional que puede competir en el mercado interno con los obtenidos a partir de otras fuentes proteicas, empleando por primera vez carne de cuy (Cavia porcellus).

\section{Materiales y métodos}

Se seleccionaron animales adultos que pesaron entre 932 y 1287 g. Se aplicó un Diseño Completamente al Azar, para lo cual se utilizaron 9 unidades experimentales, distribuidas en tres líneas genéticas (Criollo, Andino y Peruano Mejorado) procedentes de la Unidad de Especies Menores de la Facultad de Ciencias Pecuarias de la ESPOCH, la cual aplica un sistema de crianza técnico, con alimentación a base de alfalfa y desechos de cosecha, complementada con concentrado. Se tomaron 3 repeticiones por línea genética, en donde cada unidad experimental estuvo conformada por un animal.

Para ejecutar los análisis fisico-químicos y microbiológicos en la carne de cuy se preparó la muestra de acuerdo con lo que establecen las Normas Técnicas Ecuatorianas (7-8) y se realizaron los siguientes análisis, siguiendo la metodología establecida por la AOAC:

Humedad por gravimetría (9), proteína método de Kjeldahl (10), grasa por gravimetría (11), ceniza por gravimetría (12), pH (13).

Se identificaron los siguientes microorganismos: Coliformes totales (14), Escherichia coli (15), Salmonella spp. (16) y Staphylococcus aureus (17).

Caracterización sensorial: Se aplicó la técnica descriptiva para evaluar sensorialmente la carne cruda de las tres líneas de cuyes utilizadas, considerando las características organolépticas, aspecto y olor, como las más importantes, teniendo en cuenta la naturaleza del mencionado ingrediente y del proceso tecnológico por seguir, de acuerdo con los requeridos por la literatura (18). Las pruebas se realizaron en una sala de catación con las condiciones referidas por la NC ISO (19) y con la participación de 5 catadores adiestrados en este tipo de producto. 


\section{Resultados y discusión}

La Tabla I revela los resultados de los diferentes análisis fisicoquímicos realizados en la carne de cuy de las tres líneas evaluadas.

La carne de cuy Criollo presentó19,39 \% de proteína, valor que difiere estadísticamente, según Duncan $(P>0,05)$, con relación a los valores registrados en los cuyes Andinos y Peruanos mejorados, cuyos valores fueron $18,55 \%$ y $17,78 \%$, respectivamente, resultados inferiores a los reportados por los autores (20-21-22) y superiores a los obtenidos por la literatura (23); esto se debe a la influencia genética. Mientras que los mayores contenidos de grasa se registraron en la carne de los cuyes Peruanos mejorados, con un promedio de $8,56 \%$, reportándose diferencias estadísticas, según Duncan $(P>0,05)$, con relación a los valores identificados en cuyes Criollos y Andinos, que fueron de $7,93 \%$ y $7,66 \%$, respectivamente, valores superiores a los reportados por unos autores (20) y similares a los reportados por otros autores (21); esta variabilidad se presenta debido al tipo de alimentación, a la incidencia del contenido de agua y al propósito productivo; la literatura (23) cita que la carne de conejo tiene un contenido de grasa superior debido a las características propias de cada especie, alimentación y a la edad. En cuanto a la humedad, las cantidades más altas se registraron en la carne de cuyes Peruanos mejorados y Andinos, que presentaron promedios de 73,48 $\%$ y $72,83 \%$, siendo inferiores estadísticamente, según Duncan $(P>0,05)$, con respecto a la cantidad registrada en la carne de cuyes Andinos, cuyo resultado fue $75,84 \%$, valores superiores a los reportados por la bibliografía (20-22) y superiores a los reportados en la literatura en carne de conejo (21); estas diferencias se deben a la presencia de grasa en la carne, a la influencia del tipo de alimentación y a la especie animal. Con relación al contenido de ceniza, las líneas Peruano mejorado y Criollo registraron los mayores valores, con 1,26 $\%$ y $1,21 \%$, respectivamente, reportándose diferencias estadísticas, de acuerdo con el análisis de Duncan $(P>0,05)$, con relación a la carne de la línea Andino, que alcanzó un promedio de 1,08 $\%$, en lo cual influye la cantidad de minerales en la carne; la bibliografía reporta niveles superiores en carne de conejo mestizo (22). En cuanto al pH, no se encontraron diferencias estadísticas, según Duncan $(P>0,05)$, en la carne de este roedor; los valores fueron $6,47,6,41$ y 6,38 en las líneas Peruano mejorado, Criollo y Andino, respectivamente, resultados superiores a los reportados en carne de conejo de la raza Nueva Zelanda Blanco (23) e inferiores a los registrados en la literatura en carne de cuy de la raza Peruano mejorado (24).

Tabla I. Caracterización fisicoquímica de la carne de tres líneas de cuyes.

\begin{tabular}{|l|c|c|c|}
\hline \multicolumn{1}{|c|}{ Variables } & Peruano mejorado & Criollo & Andino \\
\hline Proteína \% & $17,78^{\mathrm{c}}(0,23)$ & $19,39^{\mathrm{a}}(0,25)$ & $18,55^{\mathrm{b}}(0,27)$ \\
Grasa \% & $8,56^{\mathrm{a}}(0,40)$ & $7,93^{\mathrm{ab}}(0,10)$ & $7,66^{\mathrm{b}}(0,45)$ \\
Humedad \% & $73,48^{\mathrm{c}}(0,08)$ & $72,83^{\mathrm{b}}(0,08)$ & $75,84^{\mathrm{a}}(0,06)$ \\
Ceniza \% & $1,26^{\mathrm{a}}(0,04)$ & $1,21^{\mathrm{a}}(0,03)$ & $1,08^{\mathrm{b}}(0,03)$ \\
$\mathrm{pH}$ & $6,47^{\mathrm{a}}(0,07)$ & $6,38^{\mathrm{a}}(0,04)$ & $6,41^{\mathrm{a}}(0,07)$ \\
\hline
\end{tabular}

Letras diferentes indican diferencia significativa $p<0,5$.

La Tabla II muestra la presencia de Coliformes totales, Escherichia coli, Staphylococcus aureus y Salmonella spp., microorganismos evaluados en carne de cuyes Peruanos mejorados, Criollos y An- dinos; de acuerdo con el análisis realizado, todos se encuentran dentro de los rangos de aceptación establecidos por la Norma Técnica Ecuatoriana (25), cuyos parámetros se indican en la Tabla III. 
Tabla II. Presencia de microorganismos en carne de tres líneas de cuyes.

\begin{tabular}{|l|l|c|}
\hline \multicolumn{1}{|c|}{ Microorganismo } & \multicolumn{1}{|c|}{ Líneas de cuyes } & UFC/g (log) \\
\hline \multirow{4}{*}{ Coliformes totales } & Criollo & 1,0 \\
& Peruano mejorado & 1,0 \\
Escherichia coli & Andino & 1,0 \\
& Criollo & 1,0 \\
Staphylococcus aureus & Peruano mejorado & 1,0 \\
& Andino & 1,0 \\
Salmonella spp. & Criollo & 2,5 \\
& Peruano mejorado & 2,2 \\
& Andino & 2,5 \\
& Criollo & Ausencia \\
& Peruano mejorado & Ausencia \\
\hline
\end{tabular}

Tabla III. Rangos de aceptación de la población de bacterias NTE INEN: 1346.

\begin{tabular}{|l|l|l|}
\hline \multicolumn{1}{|c|}{ Microorganismo } & \multicolumn{1}{c|}{ Mínimo } & \multicolumn{1}{c|}{ Máximo } \\
\hline Coliformes totales & $1,0 \times 10^{6}$ & $1,0 \times 10^{7}$ \\
Escherichia coli & $1,0 \times 10^{2}$ & $1,0 \times 10^{3}$ \\
Staphylococcus aureus & $1,0 \times 10^{2}$ & $5,0 \times 10^{2}$ \\
Salmonella spp. & Ausencia & \multicolumn{1}{c}{---- } \\
\hline
\end{tabular}

La Tabla IV indica los resultados obtenidos del análisis sensorial descriptivo que buscó caracterizar la carne de cuy para ser empleada en la elaboración del embutido, describiendo un color blanco de la piel, con brillo más acentuado en el cuy Andino; una masa interna de color rojo, menos intenso en los cuyes Peruano mejorado y Criollo, y un olor característico a carne fresca, propio de esta especie.

Tabla IV. Perfil sensorial descriptivo en carne de tres líneas de cuyes.

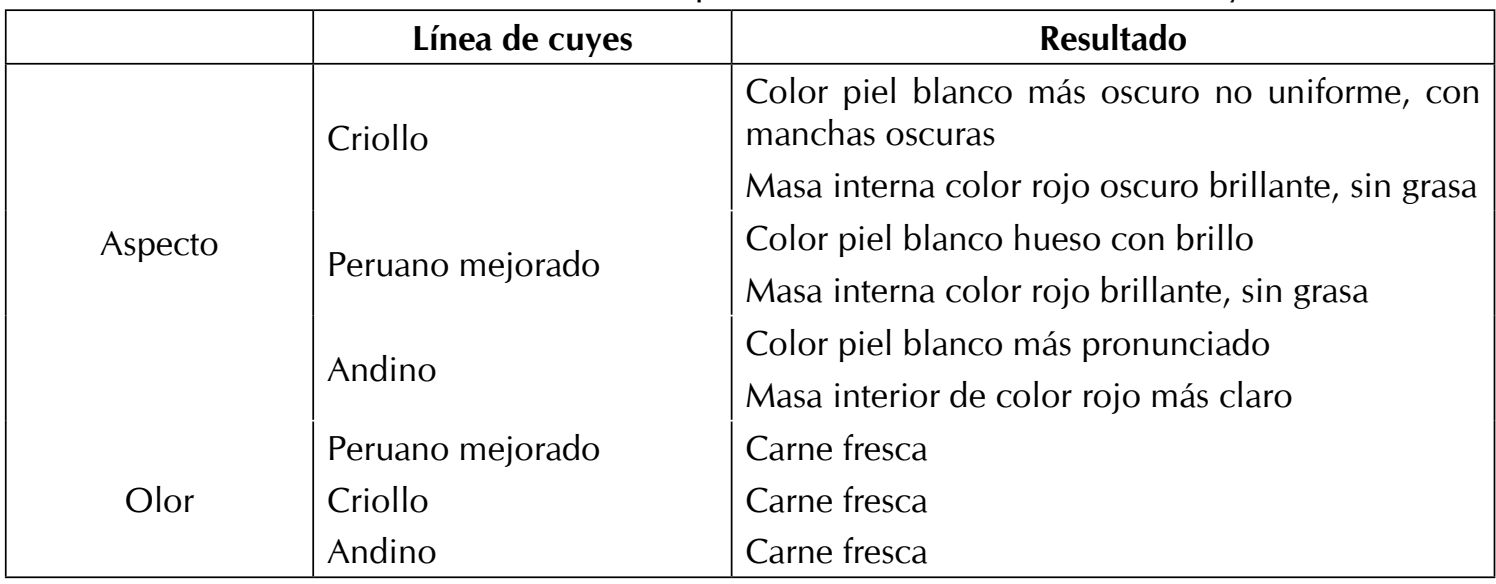




\section{Conclusiones}

El mayor contenido de proteína se presentó en carne de cuy Criollo, el más alto de grasa en el cuy Peruano mejorado, cantidades mayores de humedad, en la carne de cuyes Peruano mejorado y Andino respectivamente, los valores de $\mathrm{pH}$ no presentaron diferencias estadísticas.

La presencia de Coliformes totales, Escherichia coli, Staphylococcus aureus y Salmonella spp. en carne de cuyes Peruano mejorado, Criollo y Andino se encuentra dentro de los rangos de aceptación establecidos por la Norma Técnica Ecuatoriana 1338.

De acuerdo con la caracterización realizada se concluye que es factible utilizar la carne de las tres líneas de cuyes evaluadas para la elaboración de un embutido fermentado.

\section{Referencias}

(1) Avilés D., Landi V., Delgado J., Martínez A. El pueblo ecuatoriano y su relación con el cuy. AICA. 2014; 4: 38-40.

(2) Santos V. Importancia del cuy y su competitividad en el mercado. Arch. Latinoamérica de Producción Animal. 2007; 15(1): 216-217.

(3) Chávez S. Tecnologías de Producción y Comercialización de carne de cuy procesada para el mercado nacional y de exportación. Editorial GOYOQ. Lima, Perú. 2013.

(4) Argote F., Cuervo R. Agroindustrialización de la carne de cuy. Revista Guillermo de Ockham. 2012; 10 (2): 217-218.

(5) Calzada J. Crianza del cuy (cavia porcellus). Rvta. ACPA. 2009:19.

(6) RTE INEN: 056. Carne y productos cárnicos. Instituto Ecuatoriano de Normalización. 2013.

(7) NTE INEN: 776. Carne y productos cárnicos. Muestreo. Instituto Ecuatoriano de Normalización. 1985.

(8) NT INEN: 1529-2. Control microbiológico de los alimentos. Toma, envío y preparación de muestras para el análisis microbiológico. Instituto Ecuatoriano de Normalización. 1999
(9) AOAC. Ed 19, 925.10. Meat and meat products. Determination of humidity. Association of Official Analytical Chemists. 2012.

(10) AOAC-19 2001.11. Meat and meat products Protein determination. Association of Official Analytical Chemists. 2012.

(11) AOAC-19.991.36. Meat and meat products. Fat determination. Association of Official Analytical Chemists. 2012.

(12) AOAC-19.923.03. Meat and meat products. Determination of ash. Association of Official Analytical Chemists. 2012.

(13) NTE INEN 783:85. Carne y productos cárnicos, Determinación del $\mathrm{pH}$. Instituto Ecuatoriano de Normalización. 2012.

(14) AOAC -19. 991.14. Meat and meat products. Determination of total coliforms. Association of Official Analytical Chemists. 2012.

(15) AOAC-19. 991.14. Determination of Escherichia coli. Association of Official Analytical Chemists. 2012.

(16) AOAC-19. 998.09. Determination de Salmonella spp. Association of Official Analytical Chemists. 2012.

(17) AOAC-19. 2003.11. Determination de Staphylococcus aureus. Association of Official Analytical Chemists. 2012.

(18) Espinoza J. Evaluación sensorial de los alimentos. Editorial Universitaria, Ministerio de Educación Superior de Cuba. 2014.

(19) NC ISO: 1035. Análisis sensorial. Identificación y selección de descriptores para el establecimiento de un perfil sensorial mediante un enfoque multidimensional. Oficina Nacional de Normalización de Cuba. 2015.

(20) Apráez J., Fernández L., Hernández A. Efecto del empleo de forrajes y alimentos no convencionales sobre el comportamiento productivo, rendimiento y calidad de la carne de cuyes (Cavia porcellus). RevVet. Zootec. 2008; 2(2): 29-34.

(21) Coronado D., Parrado M. Mejorando la crianza de cuyes en Cutervo, microcuenca del Chotano. Escuela Campesina de Educación y Salud. Lima, Perú. 2010. 
(22) Malavé A., Córdova L, García A, Méndez J. Rev. MVZ Córdoba. 2013; 18(2): 3452-3458.

(23) Cury K., Martínez A. Caracterización de carne de conejo y producción de salchicha. Rev. Colombiana Ciencia Animal. 2011; 3(2): 269-282.

(24) Nakandakari L., Gutiérrez E., Chauca L., Valencia R. Medición del pH intramuscular del cuy (Cavia porcellus). Rev. Salud Tecnol. Vet. 2014; 2: 99105. DOI: http://doi.org/10.20453/stv.v2i2.2246.

(25) NTE INEN: 1346. Carne y productos cárnicos. Carne molida. Requisitos. Instituto Ecuatoriano de Normalización. 2010. 